\title{
Caracterização de lesões bucomaxilofaciais decorrentes de agressão física: diferenças entre gênero
}

\author{
Characterization of oral maxillofacial lesions resulting \\ from physical aggression: differences between genders
}

Ramiro Heleno Mesquita Garcez ${ }^{1}$

Erika Bárbara Abreu Fonseca Thomaz ${ }^{2}$

Rodrigo Campos Marques ${ }^{1}$

Juliana Aires Paiva de Azevedo ${ }^{3}$

Fernanda Ferreira Lopes ${ }^{3}$

${ }^{1}$ Graduação em

Odontologia, Centro de

Ciências Biológicas e da

Saúde (CCBS), Universidade

Federal do Maranhão

(UFMA). Av. Portugueses

1966, Vila Bacanga. 65080

805 São Luís MA Brasil.

ramirogarcez@hotmail.com

${ }^{2}$ Programa de Pós-

Graduação em Odontologia

e em Saúde Coletiva,

Departamento de Saúde

Pública, CCBS, UFMA. São

Luís MA Brasil.

${ }^{3}$ Programa de Pós-

Graduação em Odontologia,

Departamento de

Odontologia, CCBS, UFMA.

São Luís MA Brasil.

\begin{abstract}
In developed countries, violence is the main cause of oral maxillofacial (OMF) injuries. In Brazil, there are scant records of such lesions. The scope of this study was to detail the OMF injuries resulting from physical aggression in a capital of the Brazilian northeast and analyze gender differences. The Forensic Medicine Institute of São Luis, state of Maranhão, featured 15,847 reports, which occurred in 2012, and they were investigated. Socioeconomic, demographic data and OMF characteristics were analyzed. The Chi-square and Fisher's Exact tests were applied to assess gender differences. Of the cases examined, 1977 were OMF. Female victims, aged 20-59, dark-skinned, without a partner and maids were the most affected. Ecchymosis-type lesions, in the mouth and lip regions arising from the use of blunt instruments, causing permanent functional impairment, were the most prevalent in women $(P<0.05)$, whereas dental fracture and contusion arising from sharp instruments causing permanent deformity, occupational disability, and threat to life were detected in men $(P<0.05)$. The conclusion drawn is that the incidence of OMF due to physical aggression is high in São Luís, state of Maranhão, and although women are the most affected, the OMF in men are more severe.
\end{abstract}

Key words Violence, Facial trauma, Forensic dentistry
Resumo Em países desenvolvidos, a violência é a principal causa de lesões bucomaxilofaciais (LBMF). No Brasil, há poucas evidências sobre tais lesões. Objetivou-se caracterizar as LBMF resultantes de agressão física em uma capital do nordeste brasileiro e analisar diferenças entre gêneros. Foram investigados 15.847 laudos do Instituto Médico Legal de São Luís/MA, ocorridos em 2012. Coletaram-se dados socioeconômicos, demográficos e características das LBMF. Utilizaram-se os testes Qui-quadrado e Exato de Fisher para avaliar diferenças entre gêneros. Dos casos periciados, 1977 eram LBMF. Vítimas do sexo feminino, com 20-59 anos de idade, cor parda, sem companheiro e empregadas foram mais afetadas. Lesões do tipo equimose, nas regiões bucinadora e labial, decorrentes do uso de instrumentos contundentes, ocasionando debilidade funcional permanente foram mais incidentes em mulheres $(P<0,05)$, ao passo que fratura dental, ferida contusa e perfurocontusa, decorrentes de instrumentos cortantes e perfurocontundentes, ocasionando deformidade permanente, incapacidade ocupacional e perigo de vida, em homens $(P<0,05)$. Conclui-se que a incidência de LBMF decorrente de agressão física é alta em São Luís, MA, e, embora as mulheres sejam mais acometidas, as LBMF em homens são mais severas.

Palavras-chave Violência, Traumatismos faciais, Odontologia legal 


\section{Introdução}

A Organização Mundial de Saúde (OMS) define violência como o uso intencional da força física ou do poder, ameaçado ou efetivamente, contra si mesmo, outra pessoa, ou contra um grupo ou comunidade, que resulta ou tem alta probabilidade de resultar em lesão, morte, dano psicológico, mau desenvolvimento ou privação ${ }^{1}$.

A cada ano, milhares de pessoas sofrem ferimentos não fatais, como resultado de violência interpessoal ou coletiva ${ }^{2}$, resultando, muitas vezes, em lesões bucomaxilofaciais ${ }^{3}$. Na Austrália, assaltos $(74 \%)$ foram os principais motivos de lesões bucomaxilofaciais ${ }^{4}$. Em países como a Finlândia, Estados Unidos e Suécia, a violência tem sido relatada como a principal causa de injúrias faciais, o que reforça a necessidade de ampliar o conhecimento sobre a ocorrência deste agravo e de suas consequências em outros países do mun$\mathrm{do}^{5,6}$.

No Brasil, a violência física é o tipo mais frequente de violência perpetrada contra a mulher ${ }^{7,8}$ e é apontada como a primeira ${ }^{9,10}$ ou a segunda ${ }^{11-13}$ maior causa de lesões faciais. As lesões decorrentes de violência física podem atingir os tecidos moles e ocasionar fraturas nos ossos faciais ${ }^{14}$, incluindo o complexo maxilomandibular, ${ }^{3,11}$.

Estudo em 1024 vítimas de Piracicaba, São Paulo, apontou que fraturas maxilofaciais são especialmente decorrentes de acidentes de trânsito $(45 \%)$, assaltos $(22,6 \%)$, quedas $(17,9 \%)$, esportes $(7,8 \%)$ e acidentes de trabalho $(4,5 \%)$. As regiões anatômicas mais acometidas foram mandíbula (44,2\%), complexo zigomático (32,5\%) e ossos nasais $(16,2 \%)^{15}$. As mesmas regiões anatômicas foram referidas em Belo Horizonte, Minas Gerais $^{11}$. Entre os 1326 casos avaliados retrospectivamente, a maioria ocorreu em adultos (21 a 30 anos), do sexo masculino, numa razão de 4,69 homens para cada mulher ${ }^{11}$.

Homens e mulheres podem apresentar tipos específicos de lesões oriundas de agressão física $^{3,16}$. Há situações em que os homens são os agressores, vitimando suas próprias companhei$\operatorname{ras}^{17-19}$. Estudo retrospectivo na Malásia apontou que a maioria das vítimas eram mulheres casadas $(85,1 \%)$, lesionadas por seus maridos $(83,5 \%)$. Injúrias na região maxilofacial foram as mais comuns (50,4\%), especialmente no terço médio da face $(60,6 \%)$. No tecido mole, contusões, abrasões e lacerações foram as mais incidentes ${ }^{18}$. Ainda assim, a violência contra a mulher tem sido pouco evidenciada. Este problema ainda permanece velado e mal compreendido; e poucos estu- dos têm sido realizados nas regiões mais pobres do Brasil ${ }^{3,20-22}$.

O objetivo deste trabalho foi estimar a incidência de lesões no complexo bucomaxilofacial e caracterizar as lesões resultantes de violência física periciados pelo Instituto Médico Legal (IML) de uma capital do Nordeste brasileiro, analisando diferenças entre gêneros.

\section{Material e métodos}

Esta pesquisa de campo, retrospectiva, baseou-se no levantamento de dados dos laudos de exame de corpo de delito do IML da cidade de São Luís, Maranhão, ocorridos entre janeiro e dezembro de 2012.

Do total de laudos, foram incluídos os casos de lesões bucomaxilofaciais decorrentes de violência física. Foram consideradas como violência física os casos de violência interpessoal, urbana ou doméstica, que envolviam o uso de partes do corpo humano e/ou o uso de objetos com o intuito de machucar ou prejudicar a saúde da vítima. Como o exame de corpo de delito realizado no IML visa à análise de lesões corporais resultantes de crimes, os casos de lesões auto infringidas não foram incluídos. Os dados foram coletados após aprovação do estudo no Comitê de Ética em Pesquisa do Hospital Universitário da Universidade Federal do Maranhão, bem como após a concordância oficial do IML.

Os laudos foram confeccionados por ocasião dos exames de corpo de delito realizados em periciandos provenientes de Delegacias de Polícia. Quando necessário, exames eram solicitados por autoridade policial ou judicial.

A Superintendência de Polícia Técnico-Científica do Maranhão (SPTC) é vinculada à Delegacia Geral da Polícia Civil e conta com sete órgãos: IML; Instituto de Criminalística (ICRIM); Instituto de Identificação (Ident); Instituto Laboratorial de Análise Forense (ILAF); o Centro de Perícias Técnicas para a Criança e ao Adolescente (CPTCA); a Central de Custódia de Vestígios Criminais (CCVC); e o Instituto de Genética Forense (IGF). A sede destes órgãos está localizada em São Luís, capital maranhense. Por ocasião desta pesquisa, apenas outras duas cidades do estado, Imperatriz e Timon, também possuíam IML e ICRIM, porém, de menores proporções. Portanto, os casos atendidos no IML de São Luís podem ser oriundos de todo o Estado do Maranhão, mas a maioria é proveniente da capital e região metropolitana. 
A equipe do estudo consistiu de dois acadêmicos de odontologia, sob a supervisão de uma cirurgiã-dentista, especialista em Odontologia Legal. Foi previamente realizado um treinamento teórico e prático, no próprio IML, com o propósito de compreensão dos formulários e do processo de trabalho do IML, além de discussão dos instrumentos de coleta de dados. Vários laudos foram discutidos para fins de qualificação e padronização do processo de coleta. Os avaliadores acessaram os dados arquivados em sistema de informação próprio do IML de São Luís, MA. A instituição não sofreu alteração de sua rotina em decorrência desta pesquisa.

Dados dos laudos foram digitados em uma planilha previamente preparada no programa Excel 2003 for Windows ${ }^{\circledR}$. Foram consideradas as seguintes variáveis: data do exame, sexo (masculino, feminino), situação conjugal (com ou sem companheiro), idade (em anos), raça/cor (branca, preta ou outra), situação ocupacional (empregado, aposentado, estudante ou desempregado), tipo de instrumento que causou a injúria (fisicoquímico, contundente, corto contundente, cortante, perfurante ou combinações), região anatômica da lesão (cavidade oral, bucinadora, frontal, labial, malar, massetérica, mentoniana, nasal, orbitária, zigomática, hemiface e terços da face), tipo de lesão (escoriação, edema, equimose, hematoma, fratura dental, perda dental, ferida contusa, ferida perfurocontusa, ferida perfuroincisa, ferida incisa e ferida cortocontusa).

Registrou-se ainda se a agressão resultou em deformidade permanente (dano estético de certo vulto, permanente, visível, incurável e capaz de causar impressão vexatória ou sensação de repulsa no observador), debilidade permanente de função (enfraquecimento evidente, diminuição da capacidade funcional de forma permanente, duradoura, mas não necessariamente perpétua), incapacidade ocupacional (perda da capacidade para as ocupações habituais por mais de trinta dias como decorrente de qualquer atividade desenvolvida no dia-a-dia, como o trabalho, a higiene e a recreação, conforme o Código Penal brasileiro) e se a agressão causou perigo de vida (quando era provável a morte da vítima em decorrência da lesão sofrida). O termo "permanente" referiu-se à não resolução por evolução natural ou por tratamentos costumeiros. Para essas variáveis considerou-se as opções de resposta sim, talvez (quando havia sido solicitado exame complementar e os resultados ainda não estavam disponíveis) ou não. Para as análises, devido ao pequeno tamanho amostral, as categorias sim e talvez foram consideradas em conjunto.

Os dados foram submetidos à análise descritiva, estimando-se frequências absolutas e percentuais para as qualitativas. A comparação das distribuições, de acordo com características dos periciados, foi efetuada por meio dos testes Quiquadrado e Exato de Fisher, adotando-se nível de significância de $5 \%$. O software Stata, versão 12.0 (Stata Corp., College Station, TX, USA) foi utilizado.

\section{Resultados}

Foram identificados 15847 laudos nos arquivos do IML de São Luís-MA, em 2012. Para este trabalho foram incluídos 1977 laudos referentes aos casos de lesão corporal em que a vítima apresentou comprometimento bucomaxilofacial resultantes de violência física, representando $12,47 \%$ do total de laudos periciados.

A maioria das vítimas eram mulheres (56,30\%), pessoas na faixa etária de 20 a 59 anos $(85,51 \%)$, de raça/cor parda $(82,09 \%)$, que viviam sem companheiro(a) $(67,98 \%)$ e incluídos no mercado de trabalho $(70,73 \%)$.

Os tipos de lesões bucomaxilofaciais mais referidos nos laudos foram: escoriação $(39,91 \%)$, equimose $(33,49 \%)$, ferida contusa $(27,31 \%)$ e edema $(23,22 \%)$. As lesões do tipo equimose foram mais incidentes em mulheres, enquanto fratura dental, ferida contusa e perfurocontusa, em homens (Tabela 1). Houve apenas um caso de ferida perfuroincisa (não incluído na tabela).

As lesões no terço médio foram as mais frequentes $(63,13 \%)$. As regiões mais acometidas foram a orbitária $(35,91 \%)$, frontal $(26,15 \%)$ e labial (23,87\%). Diferenças entre gêneros foram verificadas nas lesões labial $(\mathrm{p}=0,008)$, bucinadora $(\mathrm{p}=0,006)$ e massetérica $(\mathrm{p}=0,050)$, todas mais prevalentes em mulheres (Tabela 2).

Os instrumentos contundentes $(95,83 \%)$ foram os mais observados, especialmente em mulheres ( $\mathrm{p}<0,001)$. Lesões provocadas por instrumentos cortantes $(p=0,021)$ e perfurocontundentes $(p=0,001)$ foram mais frequentes em homens que em mulheres (Tabela 3). Houve ainda cinco casos de lesões provocadas por veneno, três por agente fisicoquímico, três por instrumento cortocontuso e uma por perfurante (dados não mostrados). 
Tabela 1. Tipo de lesão, segundo o sexo dos periciados. São Luís, MA, Brasil, 2012.

\begin{tabular}{lccccc}
\hline \multicolumn{1}{c}{ Tipo de lesão } & \multicolumn{2}{c}{$\begin{array}{c}\text { Total } \\
(\mathbf{n = 1 9 7 7 )}\end{array}$} & $\begin{array}{c}\text { Masculino } \\
(\mathbf{n = 8 6 4})\end{array}$ & $\begin{array}{c}\text { Feminino } \\
(\mathbf{n = 1 1 1 3})\end{array}$ & $\begin{array}{c}\text { p- } \\
\text { valor }\end{array}$ \\
\cline { 2 - 5 } & $\mathbf{n}$ & $\mathbf{9}$ & $\mathbf{\%}$ & $\mathbf{\%}$ & \\
\hline Escoriação & 789 & 39,91 & 38,19 & 41,24 & $0,170^{1}$ \\
Equimose & 662 & 33,49 & 29,75 & 36,39 & $0,002^{2}$ \\
Ferida contusa & 540 & 27,31 & 31,25 & 24,26 & $0,001^{2}$ \\
Edema & 459 & 23,22 & 23,38 & 23,89 & $0,880^{1}$ \\
Hematoma & 186 & 9,41 & 8,10 & 10,42 & $0,080^{1}$ \\
Ferida cortocontusa & 74 & 3,74 & 4,51 & 3,14 & $0,112^{1}$ \\
Ferida incisa & 50 & 2,53 & 2,78 & 2,34 & $0,535^{1}$ \\
Fratura dental & 18 & 0,91 & 1,62 & 0,36 & $0,004^{2}$ \\
Ferida perfurocontusa & 13 & 0,66 & 1,16 & 0,27 & $0,022^{2}$ \\
Perda dental & 10 & 0,51 & 0,81 & 0,27 & $0,093^{1}$ \\
\hline
\end{tabular}

${ }^{1}$ Teste Qui-quadrado. ${ }^{2}$ Teste Exato de Fisher.

Algum dano à integridade corporal foi verificada em 1477 casos $(99,6 \%)$ dos laudos. Sequelas com incapacidade ocupacional ( $\operatorname{sim}=1,81 \% \mathrm{e}$ talvez $=4,69 \%)$, debilidade permanente de função $(\operatorname{sim}=1,68 \%$ e talvez $=10,35 \%)$, deformidade permanente $(\operatorname{sim}=0,72 \%$ e talvez $=10,07 \%)$ e perigo à vida ( $\operatorname{sim}=0,54 \%$ e talvez $=2,21 \%)$ foram verificadas em parte dos casos, especialmente em homens (Tabela 4).

\section{Discussão}

Nesse trabalho, a maioria das vítimas eram mulheres (56,30\%). Contrapondo-se aos nossos achados, estudos internacionais ${ }^{14,23,24}$ identificaram maior proporção de homens como vítimas de traumas maxilofacias, variando de 81,1\%, em pesquisa realizada em hospital da Índia ${ }^{23}$, a 92,9\% dos casos, em estudo conduzido na Suíça $^{24}$. Os autores justificam a predominância de homens pelo fato de serem mais propensos a atividades de risco e interações sociais violentas, frequentando bares, fazendo mais uso de drogas e dirigindo perigosamente ${ }^{16}$. Porém, estes autores consideraram todos os tipos de lesões e não somente as decorrentes de violência física como no presente trabalho.

No Brasil, um maior número de vítimas do sexo masculino também foi apontado por vários autores $^{9-12,14,25}$, variando de 51,9\% dos 774 laudos analisados em Pelotas-RS ${ }^{12}$, até $81,1 \%$, em estudo envolvendo 132 pacientes em hospital universitário de Santa Catarina9. A proporção entre homens e mulheres vítimas de fraturas faciais variou de $4,69: 1^{11}$ a $7: 1^{14}$.
Por outro lado, estudo realizado no Ceará, Nordeste do Brasil, evidenciou maioria das vítimas de lesões traumáticas orais e maxilofaciais em mulheres ${ }^{20}$, semelhante ao observado na presente pesquisa. É possível que características culturais e socioeconômicas ajudem a explicar a alta prevalência de agressões físicas em mulheres nestas pesquisas, cuja amostra foi proveniente de uma das regiões menos desenvolvidas do país. Ferreira et al. ${ }^{3}$, analisando dados provenientes de um hospital no município de Guanhães, MG, referiram que a violência física interpessoal doméstica é mais comum em vítimas do sexo feminino, ao passo que a urbana, em homens.

Neste estudo, a maioria das vítimas era adulta, seguida de adolescentes. Martins Júnior et al. ${ }^{10}$, em Blumenau-SC, constataram que 91,1\% dos traumas faciais registrados foram em indivíduos acima de 18 anos. Ferreira et al. ${ }^{3}$, em Guanhães-MG, identificaram que a violência física interpessoal, em ambas as formas (doméstica e urbana) foi maior nos adultos, de forma que $83,4 \%$ dos casos de violência doméstica e 66,3\% dos casos de violência urbana foram registrados em indivíduos com 20 ou mais anos de idade ${ }^{3}$. Similarmente, Araújo et al. ${ }^{26}$, em Belém-PA, verificaram que a faixa etária que apresentou maior quantidade de casos foi entre 18 e 44 anos; enquanto para Chiaperini et al. ${ }^{25}$, em Ribeirão Preto-SP, a etária predominante entre vítimas que procuraram o IML foi a de 26 a 30 anos.

Contrariamente aos nossos resultados, Roselino et al. ${ }^{27}$, em análise de 354 laudos periciais do IML de Ribeirão Preto-SP, verificaram que o número de casos de violência diminui de acordo com o aumento da idade: $46,33 \%$ ocorreram 
Tabela 2. Local da lesão, segundo o sexo dos periciados, São Luís, MA, Brasil, 2012.

\begin{tabular}{|c|c|c|c|c|c|}
\hline \multirow[t]{2}{*}{ Região acometida } & \multicolumn{2}{|c|}{$\begin{array}{c}\text { Total } \\
(\mathbf{n}=1977)\end{array}$} & \multirow{2}{*}{$\begin{array}{c}\begin{array}{c}\text { Masculino } \\
(\mathbf{n}=864)\end{array} \\
\%\end{array}$} & \multirow{2}{*}{$\begin{array}{c}\begin{array}{c}\text { Feminino } \\
(\mathrm{n}=1113)\end{array} \\
\%\end{array}$} & \multirow[t]{2}{*}{$\begin{array}{c}\text { p- } \\
\text { valor }\end{array}$} \\
\hline & $\mathbf{n}$ & $\%$ & & & \\
\hline Terço superior & 573 & 28,98 & 29,03 & 29,62 & $0,787^{1}$ \\
\hline Terço médio & 1248 & 63,13 & 62,45 & 63,52 & $0,646^{1}$ \\
\hline Terço inferior & 749 & 37,89 & 36,62 & 38,47 & $0,428^{1}$ \\
\hline Orbitária & 710 & 35,91 & 34,61 & 36,93 & $0,286^{1}$ \\
\hline Frontal & 517 & 26,15 & 26,97 & 25,52 & $0,466^{1}$ \\
\hline Labial & 472 & 23,87 & 20,95 & 26,15 & $0,008^{2}$ \\
\hline Malar & 321 & 16,24 & 15,51 & 16,80 & $0,440^{1}$ \\
\hline Nasal & 304 & 15,38 & 14,70 & 15,90 & $0,462^{1}$ \\
\hline Zigomática & 266 & 13,45 & 13,66 & 13,30 & $0,816^{1}$ \\
\hline Bucinadora & 193 & 9,76 & 7,64 & 11,41 & $0,006^{2}$ \\
\hline Mentoniana & 130 & 6,58 & 6,71 & 6,47 & $0,828^{1}$ \\
\hline Massetérica & 102 & 5,16 & 4,05 & 6,02 & $0,050^{1}$ \\
\hline Mandibular & 68 & 3,44 & 2,78 & 3,95 & $0,155^{1}$ \\
\hline Cavidade oral & 38 & 1,92 & 2,20 & 1,71 & $0,429^{1}$ \\
\hline Dentes & 38 & 1,92 & 2,08 & 1,17 & $0,104^{1}$ \\
\hline Maxilar & 34 & 1,72 & 1,62 & 1,80 & $0,765^{1}$ \\
\hline Hemiface & 11 & 0,56 & 0,58 & 0,54 & $0,906^{1}$ \\
\hline
\end{tabular}

${ }^{1}$ Teste Qui-quadrado. ${ }^{2}$ Teste Exato de Fisher.

entre os 18 e 30 anos de idade, enquanto $28,53 \%$ ocorreram entre 31 e 45 anos de idade.

Na cidade de São Luís, o IML não é o órgão responsável por emitir laudos de corpo de delito de vítimas abaixo de 14 anos e o faz apenas excepcionalmente aos finais de semana e feriados, quando o Centro de Perícias da Criança e do Adolescente não oferece atendimento. Dessa forma, os dados de traumas em adolescentes podem estar subestimados.

Apesar do pequeno número de adolescentes na amostra estudada, constatamos que o adulto vítima de violência doméstica ou de gênero geralmente refere ter sido uma criança vítima das mesmas condições. Estudos nacionais ${ }^{21,22} \mathrm{e}$ internacionais ${ }^{6,28}$ têm evidenciado as agressões a menores. Isso sugere que o avançar da idade apenas muda a forma de violência, mas não a erradica ${ }^{21}$.

Dentre os laudos analisados neste estudo, a raça/cor parda concentrou maior número de vítimas, sem diferenças entre gênero. Negros $(9,18 \%)$ e brancos $(8,73 \%)$ apresentaram percentual semelhante de vítimas. Esses achados corroboram os de Guimarães e Vilela ${ }^{21}$, no IML de Maceió/ $\mathrm{AL}$, onde foi observada predominância das vítimas de raça/cor parda $(71,6 \%)$, mas discordam dos de Chiaperini et al. ${ }^{25} \mathrm{em}$ mulheres examinadas no IML de Ribeirão Preto/SP, onde a maior incidência foi em brancas (76\%). Provavelmente tais diferenças se devem à distribuição desigual de brancos, pardos e pretos nas diferentes cidades do Brasil. No Maranhão, a maioria da população é parda (68,6\%), ao passo que em São Paulo, 68,4\% da população se autodeclara branca ${ }^{29}$.

A maioria das vítimas examinadas viviam sem companheiro $(67,98 \%)$, sendo $64,81 \%$ dos homens e $70,33 \%$ das mulheres. Percentual próximo foi encontrado por Rezende et al. ${ }^{30}$, em estudo no IML de Belo Horizonte-MG, quando concluíram que $63 \%$ das mulheres que sofreram lesões bucodentais por violência eram solteiras e apenas $20,4 \%$ eram casadas. Por outro lado, em estudo conduzido na Malásia, a maioria das mulheres vítimas de violência $(85,1 \%)$ eram casa$\operatorname{das}^{18}$, tendo sofrido a agressão dos próprios companheiros $(83,5 \%)$. Destas, grande parte sofreu lesões na região maxilofacial $(50,4 \%)$.

Os tipos de lesões bucomaxilofaciais mais referidas nos laudos analisados foram escoriação $(39,91 \%)$, equimose $(33,49 \%)$, ferida contusa $(27,31 \%)$ e edema $(23,22 \%)$. As fraturas dentais apresentaram baixa incidência em nosso estudo $(0,91 \%)$, assim como no trabalho de Chiaperini et al. ${ }^{25}$ e de Santos et al. ${ }^{31}$ De modo semelhante aos nossos achados, Lourenço et al. ${ }^{12}$, Cavalcanti $^{13}$ e Chiaperini et al. ${ }^{25}$ referem a escoriação como o tipo de lesão mais frequente, seguido por edemas e equimoses. Saddki et al. ${ }^{18}$ verificaram 
Tabela 3. Tipo de instrumento que ocasionou a lesão, segundo o sexo dos periciados, São Luís, MA, Brasil, 2012.

\begin{tabular}{lrrrrr}
\hline \multicolumn{1}{c}{ Tipo de instrumento } & \multicolumn{2}{c}{$\begin{array}{c}\text { Total } \\
(\mathbf{n}=\mathbf{1 9 7 7})\end{array}$} & $\begin{array}{c}\text { Masculino } \\
(\mathbf{n = 8 6 4 )}\end{array}$ & $\begin{array}{c}\text { Feminino } \\
(\mathbf{n = 1 1 1 3})\end{array}$ & $\begin{array}{c}\mathbf{p}- \\
\text { Valor }\end{array}$ \\
\cline { 2 - 6 } & $\mathbf{n}$ & $\mathbf{\%}$ & $\mathbf{\%}$ & $\mathbf{\%}$ & \\
\hline Contundente & 1426 & 95,83 & 93,30 & 97,83 & $<0,001^{1}$ \\
Cortocontundente & 55 & 3,70 & 3,65 & 3,73 & $0,937^{1}$ \\
Cortante & 51 & 3,43 & 4,72 & 2,41 & $0,021^{2}$ \\
Perfurocontundente & 19 & 1,28 & 2,14 & 0,36 & $0,001^{2}$ \\
Perfurocortante & 15 & 1,01 & 1,52 & 0,60 & $0,078^{1}$ \\
\hline
\end{tabular}

${ }^{1}$ Teste Qui-quadrado. ${ }^{2}$ Teste Exato de Fisher.

maior prevalência de contusões, escoriações e lacerações, tipos agrupados em uma mesma categoria de lesões de tecidos moles $(87,7 \%)$, percentual similar ao encontrado por Batista et al. ${ }^{32}$, em pesquisa realizada em prontuários médicos de hospital de Minas Gerais (82,4\%). Ferreira et al. ${ }^{3}$ identificaram as contusões, concussões e lacerações como as lesões maxilofaciais mais incidentes ${ }^{3}$. As divergências podem ser explicadas por diferenças metodológicas - o estudo de Ferreira et al. ${ }^{3}$ incluiu vítimas de hospital de referência para 23 municípios do Vale do Rio Doce, ao passo que a presente pesquisa considerou laudos do IML provenientes de diferentes cidades do Maranhão.

As lesões do tipo equimose foram mais incidentes em mulheres, ao passo que fratura dental, ferida contusa e perfurocontusa, em homens. Leles et al. ${ }^{33}$, em pesquisa realizada em Goiânia, relatam que as características culturais e socioeconômicas têm influência significativa nas taxas de prevalência de gênero de lesões maxilofaciais. Batista et al. ${ }^{32}$ citam ainda que a epidemiologia do trauma facial é determinada pela área geográfica, fatores demográficos e pelo período de investigação.

Os casos de fraturas ósseas referenciados nos laudos foram incluídos na variável "ferida contusa", por apresentarem quantitativo mínimo em relação às demais lesões. É compreensível que nos casos de violência física, os tecidos moles sejam geralmente mais acometidos, já que para resultar em fratura óssea, é necessário que as lesões sejam associadas a traumas de alta energia ${ }^{30}$.

Do total da amostra, a região maxilofacial mais acometida foi o terço médio da face $(63,13 \%)$, porém sem diferenças significantes entre os sexos. Esse resultado é corroborado por Leles et al. ${ }^{33}$, que afirmam que a elevada incidência das lesões maxilomandibulares nas regiões nasal, complexo zigomático e região orbital é relacionada com a posição proeminente dessas estruturas anatômicas dentro do esqueleto facial e sua maior exposição ao trauma.

A região mais citada pelos legistas em laudos de violência física foi a região orbitária (35,91\%), seguida pela região frontal $(26,15 \%)$ e região labial $(23,87 \%)$. Por outro lado, Lourenço et al. ${ }^{12}$ observaram que, das lesões faciais, os lábios foram a região mais atingida, apresentando $31,2 \%$ dos casos, seguido da região nasal $(24,8 \%)$ e região orbital $(24,5 \%)$. Um estudo multicêntrico na Europa identificou a mandíbula como a região mais frequentemente acometida por injúrias traumáticas, seguida da região orbitáriazigomática-maxilar ${ }^{6}$, porém esse estudo incluiu todo tipo de lesões externas e não apenas as decorrentes de agressão física.

Diferenças estatisticamente significantes foram observadas no acometimento da região bucinadora $(\mathrm{p}=0,006)$ e labial $(\mathrm{p}=0,008)$ entre homens e em mulheres. Em ambos os sítios, as mulheres foram mais acometidas. Isso reforça a hipótese de aumento crescente de casos de violência física em mulheres ${ }^{16}$.

O principal instrumento foi o contundente (95,83\%), seguido do cortocontundente $(3,70 \%)$ e do cortante $(3,43 \%)$. Lolli et al. ${ }^{34}$ também citaram o instrumento contundente $(93,70 \%)$ como principal instrumento de ação causador das lesões faciais. Os instrumentos contundentes $(\mathrm{p}<$ 0,001 ) foram mais comuns nas lesões em mulheres, enquanto os cortantes $(p=0,021)$ e perfurocontundentes ( $p=0,001)$, em homens.

Em 99,60\% dos casos houve dano à integridade corporal e saúde do indivíduo. Tal valor é aproximadamente o mesmo em homens e mulheres. Essa análise refere-se ao primeiro quesito oficial respondido pelos profissionais do Instituto, ocasião em que é verificado se o histórico de agressão relatado pelo periciando apresenta comprovação 
Tabela 4. Sequelas das lesões, segundo o sexo dos periciados, São Luís, MA, Brasil, 2012.

\begin{tabular}{|c|c|c|c|c|c|}
\hline \multirow{2}{*}{ Sequelas das lesões } & \multicolumn{2}{|c|}{ Total $(n=1977)$} & \multirow{2}{*}{$\begin{array}{c}\text { Masculino }(\mathrm{n}=864) \\
\%\end{array}$} & \multirow{2}{*}{$\begin{array}{c}\text { Feminino }(\mathrm{n}=1113) \\
\%\end{array}$} & \multirow{2}{*}{$\begin{array}{c}\text { p- } \\
\text { valor }^{1}\end{array}$} \\
\hline & $\mathbf{n}$ & $\%$ & & & \\
\hline Deformidade permanente & & & & & $<0,001$ \\
\hline Não & 1,745 & 89,21 & 86,05 & 91,66 & \\
\hline Talvez/Sim & 211 & 10,79 & 13,95 & 8,34 & \\
\hline Debilidade permanente de função & & & & & 0,003 \\
\hline Não & 1,772 & 90,22 & 87,97 & 91,97 & \\
\hline Talvez/Sim & 192 & 9,78 & 12,03 & 8,03 & \\
\hline Incapacidade ocupacional & & & & & 0,003 \\
\hline Não & 1,396 & 93,50 & 91,36 & 95,20 & \\
\hline Talvez/Sim & 97 & 6,50 & 8,64 & 4,80 & \\
\hline Causou perigo de vida & & & & & 0,005 \\
\hline Não & 1,453 & 97,26 & 95,92 & 98,32 & \\
\hline Talvez/Sim & 41 & 2,75 & 4,08 & 1,68 & \\
\hline
\end{tabular}

${ }^{1}$ Teste Qui-quadrado.

por vestígios físicos. Em casos raros o periciando não apresentou lesões, ou por seu relato não condizer com a verdade ou por ter sido a agressão tão leve a ponto de não deixar vestígios.

Perda da capacidade para as ocupações habituais por mais de trinta dias foi constatada em $1,81 \%$ dos casos, sendo maior em homens ( $\mathrm{p}=$ 0,003). Essa perda da capacidade se refere não apenas à capacidade laboral, mas a qualquer atividade desenvolvida no dia-a-dia do periciado. Sendo assim, observamos que a violência está associada com a perda de produtividade e o isolamento social da vítima.

A violência tem ainda profundas consequências sobre a saúde. Além das limitações funcionais, as vítimas são propensas a ter problemas psicológicos, síndromes de dor crônica, depressão e distúrbios psicossomáticos ${ }^{18}$. Isso aumenta a utilização de cuidados de saúde e serviços sociais $^{18}$.

Em 1,68\% das vítimas, as lesões causaram debilidade permanente de função, em $0,72 \%$ foi constatada deformidade permanente e as agressões causaram perigo de vida em $0,54 \%$ dos casos; todos mais frequentes em homens $(\mathrm{p}<0,05)$. Poucas investigações sobre as sequelas de tais lesões foram identificadas. Lolli et al. ${ }^{34}$ observaram que 5\% das vítimas de agressão tiveram debilidade permanente de função como resultado da lesão.

Os traumas maxilofaciais são frequentemente associados à morbidade em diferentes graus de dano físico, funcional e estético, representando um desafio para os serviços de saúde pública em todo o mundo, incluindo alto custo financeiro significativo para reparação de danos estéticos ${ }^{6}$. O rosto é a parte mais visível do corpo; contribuindo para a autoimagem de um indivíduo e sua autoestima. As razões para que um agressor tenha como alvo o rosto, são pouco conhecidas, embora seja possível deduzir que o atacante tenta minar a autoestima da vítima, seja homem ou mulher ${ }^{18}$.

Compreendendo que as ações contra a violência devem ser assumidas por toda a sociedade, sugere-se que os profissionais em formação sejam mais bem preparados para detectar indícios de violência física em seus pacientes e encorajar a denúncia. Espera-se ainda que os profissionais responsáveis pela elaboração dos laudos periciais padronizem a metodologia de coleta de informações, objetivando o registro do maior número de detalhes da agressão.

São limites deste estudo a restrição a apenas um ano de avaliação e a indisponibilidade de resultados de exames complementares para alguns casos por ocasião da coleta dos dados. Por outro lado, trata-se de um estudo representativo da população de uma capital e região metropolitana na região Nordeste, evidenciando as características das lesões bucomaxilofaciais decorrentes de agressão física em uma população em situação de vulnerabilidade socioeconômica. Este é o único IML do município, o que reduz viés de seleção, por se tratar de local de referência para a realização de perícias. Apesar de terem sido incluídos apenas os casos ocorridos em 2012, o número de casos observados teve poder para identificar diferenças entre gênero para diferentes características das lesões. 
Este é um dos poucos trabalhos sobre lesões no complexo bucomaxilfacial em vítimas de violência física no Nordeste brasileiro, e o primeiro no Maranhão incluindo a totalidade de casos periciados no maior IML do Estado durante um ano. O nordeste brasileiro é peculiar pela cultura patriarcal e altos índices de violência urbana e doméstica, o que fazem deste estudo um importante instrumento para a discussão da violência física no cenário nacional.

Conclui-se que a incidência de lesões no complexo bucomaxilofacial decorrente de violência física foi de $12,47 \%$ entre pessoas periciadas no IML de São Luís, MA, no ano de 2012. Vítimas com idade entre 20-59 anos, de cor parda, sem companheiro e que possuem emprego foram majoritariamente mais lesionadas em ambos os sexos. Escoriações foram os tipos de lesões mais incidentes. A região orbitária e o terço médio da face foram as localizações mais frequentemente acometidas por lesões em ambos os sexos. Homens e mulheres foram mais agredidos por instrumento de ação contundente causando dano à integridade corporal e à saúde da vítima quase que na totalidade dos casos. Mulheres foram mais agredidas, na proporção de 1,28:1 em relação aos homens. Há diferenças entre o tipo de lesão, instrumento causador da injúria, local acometido e consequências da lesão de acordo com o gênero. Embora as mulheres sejam mais acometidas por lesões no complexo bucomaxilofacial, os homens apresentam as lesões com aspectos mais severos.

\section{Colaboradores}

RHM Garcez, EBAF Thomaz, RC Marques, JAP Azevedo e FF Lopes contribuíram efetivamente para a realização do estudo. 


\section{Referências}

1. World Health Organization (WHO). Violence and Injury Prevention. Global Consultation on Violence and Health. Violence: a public health priority. Geneva: WHO; 1996.

2. Krug EG, Dahlberg LL, Mercy JA, Zwi AB, Lozano R. World report on violence and health. Geneva: WHO; 2002.

3. Ferreira MC, Batista AM, Ferreira FO, Ramos-Jorge ML, Marques LS. Pattern of oral-maxillofacial trauma stemming from interpersonal physical violence and determinant factors. Dent Traumatol 2014; 30(1):15-21.

4. Oberdan W, Finn B. Mandibular fractures in Far North Queensland: an ethnic comparison. ANZ J Surg 2007; 77(1-2):73-79.

5. Malara P, Malara B, Drugacz J. Characteristics of maxillofacial injuries resulting from road trafficaccidents--a 5 year review of the case records from Department of Maxillofacial Surgery in Katowice, Poland. Head \& Face Med 2006; 2(1):27.

6. Boffano P, Roccia F, Zavattero E, Dediol E, Uglešić V, Kovačič Ž, Vesnaver A, Konstantinović VS, Petrović M, Stephens J, Kanzaria A, Bhatti N, Holmes S, Pechalova PF, Bakardjiev AG, Malanchuk VA, Kopchak AV, Galteland P, Mjøen E, Skjelbred P, Koudougou C, Mouallem G, Corre P, Løes S, Lekven N, Laverick S, Gordon P, Tamme T, Akermann S, Karagozoglu KH, Kommers SC, Forouzanfar T. European Maxillofacial Trauma (EURMAT) project: a multicentre and prospective study. J Craniomaxillofac Surg 2015; 43(1):6270.

7. Waiselfisz JJ. Mapa da violência 2015: homicídio de mulheres no Brasil. Brasília: ONU/OPAS/OMS; 2015.

8. Brasil. Instituto Brasileiro de Geografia e Estatística (IBGE). Sintese de Indicadores Sociais: uma análise das condições de vida da população brasileira: 2010. Rio de Janeiro: IBGE; 2010.

9. Maliska MCS, Lima Júnior SM, Gil JN. Analysis of 185 maxillofacial fractures in the state of Santa Catarina, Brazil. Braz Oral Res 2009; 23(3):268-274.

10. Martins Júnior JC, Keim FS, Santa Helena ET. Aspectos epidemiológicos dos pacientes com traumas maxilofaciais operados no Hospital Geral de Blumenau, SC de 2004 a 2009. Intl Arch Otorhinolaryngol 2010; 14(2):192-198.

11. Chrcanovic BR, Freire-Maia B, Souza LN, Araújo VO, Abreu MHNG. Facial fractures: a 1-year retrospective study in a hospital in Belo Horizonte. Braz Oral Res 2004; 18(4):322-328.

12. Lourenço MA, Conceição LD, Lund. RG, Nascimento GG, Leite FRM. Ocorrência de trauma na região bucomaxilofacial no instituto médico legal de Pelotas/RS um estudo retrospectivo. In: 21 Congresso de Iniciação Científica da Universidade Federal de Pelotas; 2012; Pelotas.

13. Cavalcanti AL, Martins VM. Perfil epidemiológico da criança e adolescente vítimas de violência física: estudo no Departamento de Medicina Legal de Campina Grande-PB. Salusvita 2008; 27(3):341-352.

14. Ungari C, Filiaci F, Riccardi E, Rinna C, Iannetti G. Etiology and incidence of zygomatic fracture: a retrospective study related to a series of 642 patients. Eur Rev Med Pharmacol Sci 2012; 16(11):1559-1562.
15. Brasileiro BF, Passeri LA. Epidemiological analysis of maxillofacial fractures in Brazil: a 5-year prospective study. Oral Surg Oral Med Oral Pathol Oral Radiol Endod 2006; 102(1):28-34.

16. Silva CJP, Ferreira RC, Paula LPP, Haddad JPA, Moura ACM, Naves MD, Ferreira EF. Traumatismos maxilofaciais como marcadores de violência urbana: uma análise comparativa entre gêneros. Cien Saude Colet 2014; 19(1):127-136.

17. Wong JY, Choi AW, Fong DY, Wong JK, Lau CL, Kam CW. Patterns, aetiology and risk factors of intimate partner violence-related injuries to head, neck and face in Chinese women. BMC Womens Health [periódico na Internet]. 2014 [acessado $2017 \mathrm{Fev} 12$ ];14(6): [cerca de 9 p.]. Disponível em: https://www.ncbi.nlm.nih.gov/ pmc/articles/PMC3893604/pdf/1472-6874-14-6.pdf

18. Saddki N, Suwaidi AA, Daud R. Maxillofacial injuries associated with intimate partner violence in women. BMC Public Health [periódico na Internet]. 2010 [acessado $2017 \mathrm{Fev}$ 12];10(268): [cerca de 8 p.]. Disponível em: http://www.biomedcentral.com/1471 2458/10/268.

19. Arosarena OA, Fritsch TA, Hsueh Y, Aynehchi B, Haug R. Maxillofacial injuries and violence against women. Arch Facial Plast Surg 2009; 11(1):48-52.

20. Carvalho Filho MAM, Saintrain MVL, Anjos RES, Pinheiro RS, Cardoso LCP, Moizan JAH, Aguiar AS. Prevalence of oral and maxillofacial trauma in elders admitted to a reference hospital in Northeastern Brazil. Plos One [periódico na Internet]. 2015 [acessado 2017 Fev 22];10(8):e0135813 [cerca de 11 p.]. Disponível em: http://journals.plos.org/plosone/article/file?id = 10.1371/journal.pone.0135813\&type $=$ printable

21. Guimarães JATL, Villela WV. Características da violência física e sexual contra crianças e adolescentes atendidos no IML de Maceió, Alagoas, Brasil. Cad Saude Publica 2011; 27(8):1647-1653.

22. Cavalcanti AL. Lesões no complexo maxilofacial em vítimas de violência no ambiente escolar. Cien Saude Colet 2009; 14(5):1835-1842.

23. Bali R, Sharma P, Garg A, Dhillon G. A comprehensive study on maxillofacial trauma conducted in Yamunanagar, India. J Inj Violence Res 2013; 5(2):108-116.

24. Businger AP, Krebs J, Schaller B, Zimmermann H, Exadaktylos AK. Cranio-maxillofacial injuries in victims of interpersonal violence. Swiss Med Wkly [periódico na Internet]. 2012 [acessado $2017 \mathrm{Fev} 22$ ];142:w13687 [cerca de 11 p.]. Disponível em: https://smw.ch/article/ doi/smw.2012.13687

25. Chiaperini A, Bérgamo AL, Bregagnolo LA, Bregagnolo JC, Watanabe MGC, Silva RHA. Danos bucomaxilofaciais em mulheres: registros do Instituto Médico-legal de Ribeirão Preto (SP), no período de 1998 a 2002. Rev Odonto Ciênc 2009; 24(1):71-76.

26. Araújo RJG, Mendes MBBL, Castro TM, Moreira Júnior MT, Araújo ABL, Pacheco LM. Análise dos traumas de face que acometem mulheres vítimas de violência doméstica. Full Dent Sci 2011; 3(9):78-85. 
27. Roselino LMR, Bregagnolo LA, Pardinho MABS, Chiaperini A, Bérgamo AL, Santi LN, Bregagnolo JC, Watanabe MGC, Silva RHA. Danos buco-maxilo-faciais em homens da região de Ribeirão Preto (SP) entre 1998 e 2002. Odonto Ciênc Saúde - Rev CROMG 2009; 10(2):71-77.

28. Boffano P, Roccia F, Zavattero E, Dediol E, Uglešić V, Kovačič Ž, Vesnaver A, Konstantinović VS, Petrović M, Stephens J, Kanzaria A, Bhatti N, Holmes S, Pechalova PF, Bakardjiev AG, Malanchuk VA, Kopchak AV, Galteland P, Mjøen E, Skjelbred P, Grimaud F, Fauvel F, Longis J, Corre P, Løes S, Lekven N, Laverick S, Gordon P, Tamme T, Akermann S, Karagozoglu KH, Kommers SC, Meijer B, Forouzanfar T. European Maxillofacial Trauma (EURMAT) in children: a multicenter and prospective study. Oral Surg Oral Med Oral Pathol Oral Radiol 2015; 119(5):499-504.

29. Instituto Brasileiro de Geografia e Estatística (IBGE). Censo demográfico 2010. Brasília: IBGE; 2010.

30. Rezende EJC, Araújo TM, Moraes MAS, Santana JSS, Radicchi R. Lesões buco-dentais em mulheres em situação de violência: um estudo piloto de casos periciados no IML de Belo Horizonte, MG. Rev Bras Epidemiol 2007; 10(2):202-214.

31. Santos SE, Marchiori EC, Soares AJ, Asprino L, de Souza Filho FJ, de Moraes M, Moreira RW. A 9-year retrospective study of dental trauma in Piracicaba and neighboring regions in the State of São Paulo, Brazil. $J$ Oral Maxillofac Surg 2010; 68(8):1826-1832.

32. Batista AM, Ferreira FO, Marques LS, Ramos-Jorge ML, Ferreira MC. Risk factors associated with facial fractures. Braz Oral Res 2012; 26(2):119-125.

33. Leles JLR, Santos EJ, Jorge FD, Silva ET, Leles CR. Risk factors for maxillofacial injuries in a Brazilian emergency hospital sample. J Appl Oral Sci 2010; 18(1):2329.

34. Lolli LF, Antunes TCG, Lolli MCGS, Lolli HA, Oliveira RMG, Neto Filho MA, Pesarini A. Perfil de violência com acometimento facial na região de abrangência do Instituto Médico Legal de Maringá no ano de 2010.

Uningá Review 2012; 10(1):58-66.

Artigo apresentado em 14/09/2016

Aprovado em 01/06/2017

Versão final apresentada em 03/06/2017 